\title{
Stress tensor and bulk viscosity in relativistic nuclear collisions
}

\author{
Rainer J. Fries, ${ }^{1,2,3}$ Berndt Müller, ${ }^{3,4}$ and Andreas Schäfer ${ }^{3,5}$ \\ ${ }^{1}$ Cyclotron Institute and Department of Physics, Texas A\&M University, College Station, Texas 77843, USA \\ ${ }^{2}$ RIKEN/BNL Research Center, Brookhaven National Laboratory, Upton, New York 11973, USA \\ ${ }^{3}$ Yukawa Institute of Theoretical Physics, Kyoto University, Kyoto 606-8502, Japan \\ ${ }^{4}$ Department of Physics, Duke University, Durham, North Carolina 27708, USA \\ ${ }^{5}$ Institut für Theoretische Physik, Universität Regensburg, D-93040 Regensburg, Germany
}

(Received 30 July 2008; published 26 September 2008)

\begin{abstract}
We discuss the influence of different initial conditions for the stress tensor and the effect of bulk viscosity on the expansion and cooling of the fireball created in relativistic heavy ion collisions. In particular, we explore the evolution of longitudinal and transverse components of the pressure and the extent of dissipative entropy production in the one-dimensional, boost-invariant hydrodynamic model. We find that a bulk viscosity consistent with recent estimates from lattice QCD further slows the equilibration of the system; however, it does not significantly increase the entropy produced.
\end{abstract}

DOI: 10.1103/PhysRevC.78.034913

PACS number(s): 25.75.Ag, 25.75.Ld, 47.75.+f

\section{INTRODUCTION}

The formation of strongly interacting matter at supranuclear energy densities has recently been studied in great detail in nuclear reactions at the Relativistic Heavy Ion Collider (RHIC). The analysis of the data collected in these experiments [1-4] has shown that the matter flows very rapidly at the moment of its breakup into free-streaming hadrons. The collective flow also exhibits a large anisotropy in noncentral collisions, characterized by the "elliptic" flow parameter $v_{2}$. These observations are commonly understood to imply that (i) the quark and gluon matter undergoes rapid equilibration with thermalization times smaller than $1 \mathrm{fm} / c$ [5], and (ii) the quarkgluon plasma (QGP) maintains a low shear viscosity $\eta$ not much larger than the Kovtun-Son-Starinets (KSS) conjectured lower bound $4 \pi \eta_{\mathrm{KSS}}=s$, where $s$ is the entropy density [6]. This has led to the claim that the quark-gluon plasma formed at RHIC is the most perfect liquid known in nature [7].

The emerging picture still has some uncertainties. The predictions of hydrodynamic simulations, especially for the flow anisotropy in off-central collisions, depend on the assumed transverse density profile [8]. Moreover, the possible role of contributions to transverse flow from the preequilibrium phase of the reaction is not settled. Most hydrodynamic calculations assume that no transverse flow is present at the time of initialization, usually chosen in the range $\tau=0.5-1.0 \mathrm{fm} / \mathrm{c}$ after the onset of the reaction, although there are good reasons to believe that transverse pressure gradients existing at earlier times will contribute to the generation of collective flow even if the parton momentum distribution is still anisotropic [9].

Recently, it was pointed out that further complications could come from the bulk viscosity $\zeta$ of quark and gluon matter near the QCD phase transition [10,11]. Bulk viscosity can be neglected compared to shear viscosity in many systems in nature. This was also shown to be true in quantum chromodynamics (QCD) at high temperature and weak coupling where $\zeta \sim \alpha_{s}^{2} T^{3} / \ln \alpha_{s}^{-1}$ [12], while $\eta \sim T^{3} /\left(\alpha_{s}^{2} \ln \alpha_{s}^{-1}\right)$ [13]. The difference can be traced back to the near conformal invariance of QCD at high temperature. Near the pseudocritical temperature $T_{c}$, however, QCD is far from being conformal, as can be inferred from the large peak of the "interaction measure" $(\epsilon-3 P) / T^{4}$ at $T_{c}$ found in lattice QCD simulations [14]. Indeed, the ratio of the bulk viscosity over the entropy density, $\zeta / s$, on the lattice (in the quenched approximation) was recently found [15] to exhibit a narrow peak around $T_{c}$ of order unity with $\zeta / s \gg \eta / s$. The bulk viscosity can also be related directly to the trace anomaly of the energy momentum tensor, and an estimate can thereby be obtained from lattice results for the interaction measure [11].

The large spike of the bulk viscosity near $T_{c}$ immediately raises several questions [11] which we want to address here. However, we also want to take a look at the larger picture. Ideal hydrodynamics requires complete thermalization of the matter. Isotropization of the pressure $P$ and consistency with the equation of state $P(\epsilon)$ are necessary conditions for equilibrium. Viscous hydrodynamics permits certain kinds of small deviations from equilibrium. In the local rest frame, shear stress $\pi^{i j}$ describes deviations from isotropy of the stress tensor, whereas bulk stress $\Pi \delta^{i j}$ measures deviations from the equilibrium equation of state. In the second-order formulation of viscous hydrodynamics [16,17], the deviations of the stress tensor from its equilibrium form are not prescribed by external strains, such as flow shear or flow divergence, but can be independently given and only relax to the externally forced values over time. The fireball produced in a relativistic nuclear collision starts out with highly anisotropic particle distributions and therefore a large pressure anisotropy, which may not be related to the imprinted flow field. It is thus useful to explore how the hydrodynamic evolution responds to different initial deviations from local equilibrium.

It is not always obvious how large the deviations can be before the hydrodynamic approximation fails. In principle, viscous corrections extend the reach of hydrodynamics in relativistic heavy ion collisions to earlier times, but the gradient expansion of the stress tensor may break down [18]. Similarly, a large bulk viscosity $\zeta$ around $T_{c}$ could mean that the matter is driven far from equilibrium around the phase transition and may even develop dynamical instabilities [19]. It has also been claimed [11] that bulk stress could contribute significantly to 
entropy production, with possibly profound consequences for the hadronization mechanism at $T_{c}$. Furthermore, one expects the viscous bulk pressure $\Pi$ to be negative for an expanding system $\left(\Pi=-\zeta \partial_{\mu} u^{\mu}\right.$ to first order in gradients), implying that the effect of bulk viscosity is to slow down the expansion of the system. This should be most relevant in the longitudinal direction, where the pressure is also reduced by the viscous shear stress. Hence, one expects the system to spend more time around $T_{c}$ than predicted by ideal hydrodynamics. This leads to yet another interesting prospect: perhaps the evolution of the system is more sensitive to the equation of state near $T_{c}$ and the order of the phase transition than one would expect from ideal hydrodynamics where the relation of the phase transition to observables has been found to be rather obscure [20].

Here, we want to explore these questions in a hydrodynamic model with a simple space-time structure, but realistic equation of state, and bulk and shear viscosities motivated by lattice QCD. Our framework is a one-dimensional boost-invariant fireball with translational and rotational symmetries in the transverse plane as first described by Bjorken [21]. We discuss the time evolution of the components of the pressure using second-order hydrodynamics. Our treatment is comparable to that of Baym [22] and Heiselberg and Wang [23,24], who explored deviations from, and the approach to, equilibrium in a boost-invariant expansion in the framework of the relaxation time approximation to the Boltzmann equation. We will also explore the consequences of several different assumptions about the initial longitudinal and transverse pressure.

Let us rephrase our catalog of questions in the context of our hydrodynamic model: (i) How far from the equilibrium pressure $P$ is the longitudinal pressure $P_{z}$ throughout the evolution and in particular at the phase transition? (ii) How close to isotropy is the pressure tensor, i.e., how large is $\left|P_{\perp}-P_{z}\right| / P$ ? (iii) What is the amount of entropy $S_{\Pi}$ produced by the bulk viscosity compared with contributions from shear viscosity $S_{\Phi}$ during the lifetime of the fireball?

We are aware that the simplified treatment with onedimensional expansion has several limitations. This approximation does not permit us to explore effects related to the transverse expansion and the more rapid cooling coming from it. We also cannot treat the flow anisotropy in the transverse plane seen in off-central collisions. Since boost-invariant hydrodynamics tends to overestimate the time required for the matter to cool below $T_{c}$, it makes our study conservative in the sense that the effects of the bulk viscosity are underestimated, compared with those expected in a full three-dimensional treatment.

\section{VISCOUS HYDRODYNAMICS}

In the center of the fireball in a nuclear collision, the viscous stress-energy tensor in the local comoving frame has the form [25-27]

$$
T^{\mu \nu}=\left(\begin{array}{cccc}
\varepsilon & 0 & 0 & 0 \\
0 & P_{\perp} & 0 & 0 \\
0 & 0 & P_{\perp} & 0 \\
0 & 0 & 0 & P_{z}
\end{array}\right),
$$

with the transverse and longitudinal pressure

$$
\begin{gathered}
P_{\perp}=P+\Pi+\frac{1}{2} \Phi, \\
P_{z}=P+\Pi-\Phi .
\end{gathered}
$$

Here $P$ denotes the (isotropic) pressure in thermal equilibrium, $\Phi$ and $\Pi$ denote the nonequilibrium contributions to the pressure coming from shear and bulk stress. In particular, the traceless shear tensor in that frame takes the form $\pi^{i j}=$ $\operatorname{diag}(\Phi / 2, \Phi / 2,-\Phi)$ consistent with the symmetries in the transverse directions. We refer the reader to Refs. [26-28] for more details.

At early times, $\Phi$ and $\Pi$ will be given by the initial conditions for the stress tensor established by the mechanisms of energy and momentum deposition in the nuclear collision. Reflecting the dilution effect of the expansion on the local longitudinal momentum distribution of partons, the pressure components are expected to satisfy the ordering

$$
P_{z} \equiv T^{z z}<T^{x x}=T^{y y} \equiv P_{\perp} .
$$

One can argue that the largest physically meaningful value of $\Phi$ at a time $\tau$ is $\Phi=4(P+\Pi)$, which corresponds to $P_{z}=-P_{\perp}$. Such a negative value of the longitudinal pressure arises, for example, when the matter is completely in the form of coherent longitudinal fields at very early times [9,29]. However, such a configuration is very far from equilibrium, and the hydrodynamic approximation is surely invalid. Once decoherence of the field is reached at a time $\tau_{\mathrm{dec}}$, the components of the physical pressure should be positive: $P_{z}, P_{\perp} \geqslant 0$. We want to explore various scenarios for the time evolution of $\Phi$ and $\Pi$, assuming initial values satisfying $\Phi \leqslant P+\Pi$ at the start time of the hydrodynamic evolution $\tau_{0} \geqslant \tau_{\mathrm{dec}}$.

In first-order (Navier-Stokes) dissipative hydrodynamics, the bulk and shear stresses are related to gradients in the system via the bulk and shear viscosities through

$$
\Pi=-\zeta \partial^{\mu} u_{\mu}, \quad \pi^{\mu \nu}=2 \eta \nabla^{\langle\mu} u^{\nu\rangle},
$$

where $u^{\mu}=(\cosh \eta, 0,0 \sinh \eta)$ in our case is the expansion velocity, $\eta$ is the space-time rapidity, $\nabla^{\mu}=\partial^{\mu}-u^{\mu}\left(u_{\nu} \partial^{\nu}\right)$, and $\langle\ldots\rangle$ indicates a projection orthogonal to $u^{\mu}$, symmetrization of indices, and removal of the trace.

We follow the spirit of the (Israel-Stewart) theory of secondorder dissipative hydrodynamics [17] by assuming that the actual bulk and shear stress have the freedom to relax to their first-order values at rates governed by relaxation times $\tau_{\Pi}$ and $\tau_{\pi}$. The equations governing the longitudinal expansion of the medium in our case are then given by [28,30-32]

$$
\begin{aligned}
\frac{\partial \varepsilon}{\partial \tau} & =-\frac{1}{\tau}(\varepsilon+P+\Pi-\Phi), \\
\tau_{\pi} \frac{\partial \Phi}{\partial \tau} & =\frac{4 \eta}{3 \tau}-\Phi(\tau)-\left[\frac{4 \tau_{\pi}}{3 \tau} \Phi+\frac{\lambda_{1}}{2 \eta^{2}} \Phi^{2}\right], \\
\tau_{\Pi} \frac{\partial \Pi}{\partial \tau} & =-\frac{\zeta}{\tau}-\Pi(\tau) .
\end{aligned}
$$

It was recently pointed out by Baier et al. [32] that the terms in the square bracket in Eq. (6) are required in a theory with conformal symmetry. Conformal symmetry is approximately realized in QCD at high temperatures. Since these terms have 
not been studied quantitatively, we will examine their influence on the evolution below.

The entropy density $s$ obeys the equation [33]

$$
\frac{\partial(\tau s)}{\partial \tau}=\frac{\tau}{T}\left(\frac{3 \Phi^{2}}{4 \eta}+\frac{\Pi^{2}}{\zeta}\right) .
$$

$\tau s=d S /(d y d A)$ is the entropy per unit rapidity $y$ and transverse area $A$. $\tau s$ is constant for ideal hydrodynamics. Bulk and shear stresses relax toward their Navier-Stokes values. The late time behavior of these values for the one-dimensional boost-invariant expansion is then directly given by Eq. (4) as

$$
\Phi=\frac{4 \eta}{3 \tau}, \quad \Pi=-\frac{\zeta}{\tau} .
$$

The larger the shear viscosity, the more anisotropic the pressure remains at late times.

Relaxation times and viscosities are related by coefficients $\beta_{0}$ and $\beta_{2}$ which are determined by the underlying theory:

$$
\tau_{\Pi}=\zeta \beta_{0}, \quad \tau_{\pi}=2 \eta \beta_{2} .
$$

Kinetic theory of massless partons predicts a value, $\beta_{2}=$ $3 /(4 P)[30,31]$. This leads to a relaxation time which is roughly given by

$$
\tau_{\pi}^{(\mathrm{kin})}=\frac{3}{2 \pi T} .
$$

In conformal hydrodynamics, a different behavior is obtained by matching the asymptotic form of a boost-invariant, longitudinally expanding thermal medium in the $N=4$ supersymmetric Yang-Mills (SYM) theory to a hydrodynamic evolution $[32,34]$

$$
\tau_{\pi}^{(\mathrm{SYM})}=\frac{2-\ln 2}{2 \pi T} .
$$

Obviously, the value of the relaxation time from kinetic theory is about twice as long as the latter. We will test both values below. In absence of further reliable predictions, we will always assume the same relaxation time for the bulk stress as a function of temperature, $\tau_{\Pi}(T)=\tau_{\pi}(T)$. The coefficient $\lambda_{1}$ in Eq. (6) was determined for supersymmetric Yang-Mills theory to be [32]

$$
\lambda_{1}=\frac{\eta}{2 \pi T}
$$

\section{VISCOSITIES AND INITIAL CONDITIONS}

We have already specified the equations of motion and our choice for the parameters $\tau_{\pi}, \tau_{\Pi}$ in the previous section. We further assume that the matter is characterized by a minimal shear viscosity, i.e., we set $\eta=\eta_{\mathrm{KSS}}=s /(4 \pi)$. This is not in contradiction to lattice QCD results [35], which lie close to the KSS bound. The pressure anisotropies found in our calculation can therefore be considered as a lower bound. For the equilibrium equation of state, we use recent lattice QCD results for one heavy and two light quark flavors [14]. We have parametrized the reduced equilibrium pressure $P / T^{4}$ and interaction measure $(\varepsilon-3 P) / T^{4}$ for $N_{\tau}=6$ lattices from this reference. The critical temperature is $T_{c}=196 \mathrm{MeV}$.
For the bulk viscosity, we explore several options. Our starting point is a recent calculation by Meyer in quenched QCD [15]. This calculation is not directly compatible with our equation of state from unquenched QCD. To deal with this problem, we have here chosen to parametrize the dimensionless ratio $\zeta / s$ as a function of the dimensionless ratio $\omega=(\varepsilon-3 P) /(\varepsilon+P)$. This choice corrects for the shift in the critical temperature, and it softens the very steeply peaked behavior of $\zeta / s$ found by Meyer in quenched QCD. We call the bulk viscosity resulting from this fit $\zeta_{0}$. We note that several mechanisms can contribute to the bulk viscosity [36]. In QCD, $\zeta / s$ is not only a function of the interaction measure but also of the speed of sound, the quark condensates, and the relaxation time scale of the compression mode [11,37]. We also note that the statistical and systematic uncertainties of the existing lattice results for quenched QCD are quite large. We account for these combined uncertainties by running the hydrodynamic evolution for several values of $\zeta$ which have been obtained from $\zeta_{0}$ by multiplying with a scaling factor $c_{\zeta}$, that is, $\zeta=c_{\zeta} \zeta_{0}$, and by changing the width of the peak near $T_{c}$ by a scale factor $1 / a_{\zeta}$.

We show the results of our fits in Fig. 1. The kinematic bulk viscosity fitted from the lattice results as a function of temperature [shown by the solid (blue) curve] is compared with the kinematic shear viscosity from the KSS relation $\eta / s=$ $1 /(4 \pi)$ [dash-dotted (black) line]. The dashed (blue) curve shows a modified parametrization for $\zeta / s$ with double the peak height and half the peak width $\left(c_{\zeta}=a_{\zeta}=2\right)$. The bulk viscosity exceeds the shear viscosity for temperatures below $220 \mathrm{MeV}$. The insert shows the interaction measure fitted from the results of Cheng et al. [14].

For all simulations, we choose a starting time $\tau_{0}=$ $0.3 \mathrm{fm} / c$ which is smaller than the equilibration times estimated from ideal hydrodynamics and is compatible with the expected decoherence time $[38,39]$ of the initial gluon field. The initial

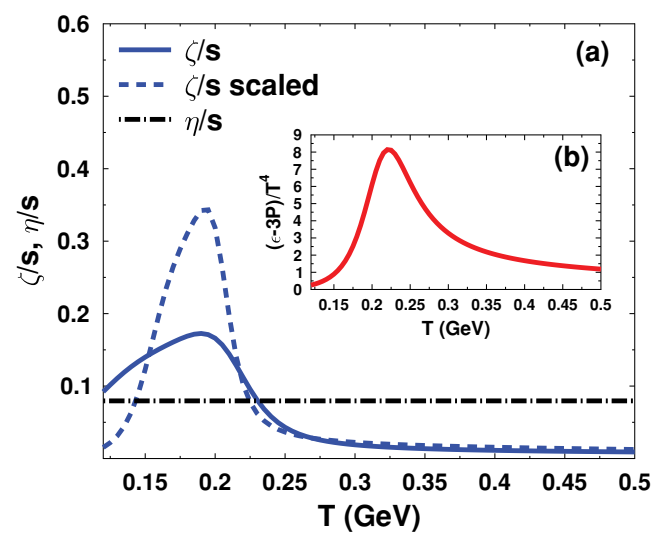

FIG. 1. (Color online) (a) Kinematic bulk viscosity $\zeta / s$ and kinematic shear viscosity $\eta / s$ as functions of temperature $T . \eta / s$ is determined by the KSS bound $\eta / s=1 /(4 \pi)$; and $\zeta / s$ is derived by a fit to the results reported in Ref. [15], using the conformal measure $\omega$ as scaling variable. This fit is denoted as $c_{\zeta}=a_{\zeta}=1$. The blue dashed curve shows a modified parametrization for $\zeta / s$ with double the peak height $\left(c_{\zeta}=2\right)$ and half the peak width $\left(a_{\zeta}=2\right)$. (b) Parametrization of the reduced interaction measure $(\epsilon-3 P) / T^{4}$ from Ref. [14] as a function of $T$. 
energy density is fixed to be $\epsilon\left(\tau_{0}\right)=50 \mathrm{GeV} / \mathrm{fm}^{3}$ which corresponds to an initial equilibrium temperature of roughly $400 \mathrm{MeV}$. We discuss three different initial conditions:

(i) Equilibration sets $\Pi\left(\tau_{0}\right)=\Phi\left(\tau_{0}\right)=0$.

(ii) First order uses the values given by first-order viscous hydrodynamics, $\Pi\left(\tau_{0}\right)=-\zeta\left(T_{0}\right) / \tau_{0}$ and $\Phi\left(\tau_{0}\right)=$ $4 \eta\left(T_{0}\right) /\left(3 \tau_{0}\right)$, where $T_{0}$ is the initial temperature at $\tau_{0}$.

(iii) Anisotropic uses $\Pi\left(\tau_{0}\right)=-\zeta\left(T_{0}\right) / \tau_{0}$ as in condition (ii), but fixes $\Phi\left(\tau_{0}\right)=P\left(\tau_{0}\right)+\Pi\left(\tau_{0}\right)$ to set the initial longitudinal pressure to zero.

We also remind the reader that we will run the hydrodynamic evolution both with the conformal terms in Eq. (6) (we will denote this scenario by the label "C") and in a standard version without them (denoted by "S"). We also explore both the short ("SYM") and long ("kin") relaxation time. Each run below will be denoted by a four-component label indicating the set of initial conditions, relaxation times, absence or presence of the conformal terms, and the scaling variable $c_{\zeta}=\zeta / \zeta_{0}$.

\section{RESULTS}

In this section, we present the results of numerical solutions of the viscous hydrodynamic Eqs. (5)-(7). We start by checking the influence of different values of the bulk viscosity by varying $c_{\zeta}$ with $a_{\zeta}$ fixed. We compare the vanishing bulk viscosity $\left(c_{\zeta}=0\right)$ with the lattice inspired value $\left(c_{\zeta}=1\right)$. For these runs, we fix the set of initial conditions to anisotropic (iii), use evolution without the conformal terms (S), and choose the short relaxation time (SYM). Figure 2 shows the development of different components of the pressure relative to the equilibrium pressure $P$ as a function of time. Figure 2(a) shows the dissipative stress components $-\Pi / P$ and $\Phi / P$ due to bulk and shear viscosity, respectively, for $c_{\zeta}=1$. As expected, the effects of shear viscosity are dominant at early times, while bulk stress peaks later, when $\zeta / s$ starts to rise around $T_{c}$. The peak in $-\Pi / P$ is reached already slightly before the critical temperature $T_{c}$; the time $\tau_{c} \approx 8.4 \mathrm{fm} / c$ when the system reaches $T_{c}$ (for $c_{\zeta}=1$ ) is indicated by the solid triangles on the abscissae.

Figure 2(b) displays the relative longitudinal and transverse pressure $P_{\perp} / P$ and $P_{z} / P$, respectively, for $c_{\zeta}=1$ (solid lines) and $c_{\zeta}=0$ (dashed lines). Both quantities develop a pronounced minimum just above $T_{c}$ if bulk viscosity is present, which corresponds to the maximum in $-\Pi / P$. The minima above $T_{c}$ are absent for vanishing bulk viscosity. We observe that the system is still very anisotropic even at $T_{c}$; and with $c_{\zeta}=1$, both pressure components are well below the equilibrium value.

As mentioned earlier, the stability of boost invariant relativistic hydrodynamics in the presence of a bulk viscosity was recently studied by Torrieri and Mishustin [19]. Their treatment differs from ours in two important details: they study the first-order (Navier-Stokes) formulation of viscous hydrodynamics, and they use a parametrization of the temperature dependence of the bulk viscosity which is much more strongly peaked near $T_{c}$ and attains a much higher peak value. Even for their most conservative choice $\left(z_{0}=0.1\right.$ in the notation of

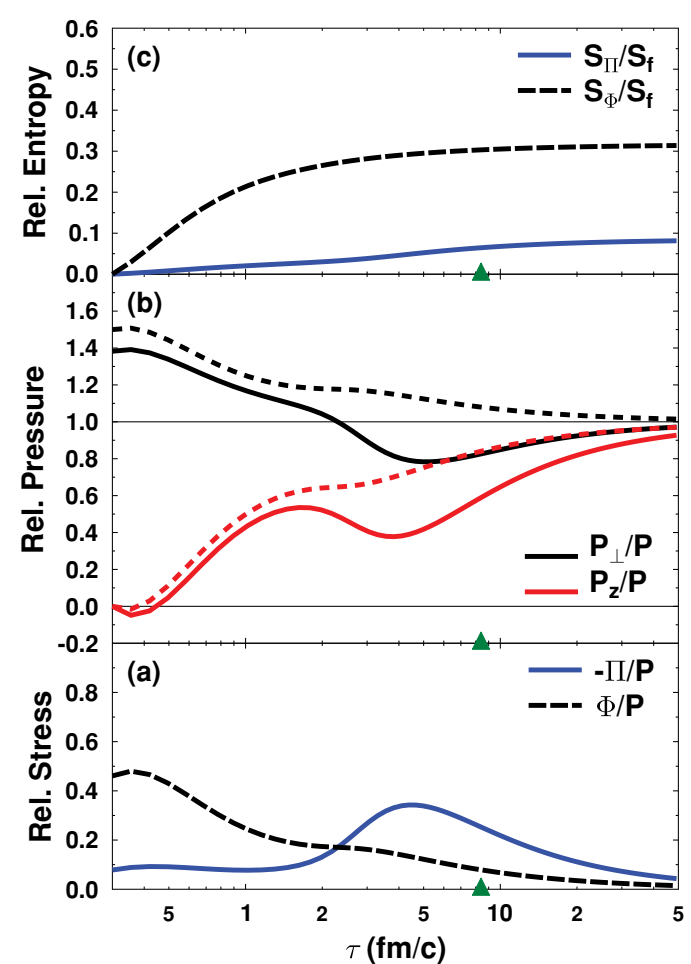

FIG. 2. (Color online) (a) Relative bulk and shear stresses, $-\Pi / P$ and $\Phi / P$, as functions of time $\tau$ for the scenario (iii, S, SYM, $c_{\zeta}=1$ ). The time when $T_{c}$ is reached is indicated by the triangle. (b) Relative transverse and longitudinal pressures, $P_{\perp} / P$ and $P_{z} / P$, as functions of time $\tau$ for (iii, S, SYM, $c_{\zeta}=1$ ) (solid lines) and for the same scenario but with $c_{\zeta}$ set to zero (dashed lines). (c) Relative entropy production from bulk and shear stress, $S_{\Pi} / S_{f}$ and $S_{\Phi} / S_{f}$, as functions of time $\tau$ for the same scenarios with $c_{\zeta}=1$. All results are for $a_{\zeta}=1$.

Ref. [19]), the peak in $\zeta / s$ is about 20 times higher than our peak value and 5 times as high as the largest value obtained by Meyer [35]. We have solved our set of equations with their parameters and used a very small relaxation time $\tau_{\Pi}=\tau_{\pi}$ to emulate the Navier-Stokes limit. We find that $P_{z}$ becomes strongly negative in the range where $\zeta / s$ peaks, indicating that the matter is not only hydrodynamically unstable, as found in Ref. [19], but also thermodynamically unstable. This behavior is obviously a result of the highly peaked parametrization adopted in Ref. [19].

We now return to our own study. Figure 2(c) shows the entropy per unit rapidity and transverse area produced by shear and bulk viscous effects, $S_{\Phi}=\tau s_{\Phi}$ and $S_{\pi}=\tau s_{\Pi}$, respectively. They correspond to the first and second terms in Eq. (8) and are shown relative to the total final value $S_{f}=$ $\tau_{f} s\left(\tau_{f}\right)$, where the final time $\tau_{f}$ is fixed at $50 \mathrm{fm} / c$. We note that despite its dramatic effects on the longitudinal pressure, the contribution of the bulk stress to entropy production is rather moderate. The entropy produced by shear stress is much larger because of the large velocity gradient in the initial state which generates large dissipative effects. However, the majority of the entropy production is confined to the earliest time period $\tau_{0}<\tau<1 \mathrm{fm} / c$, suggesting that a hydrodynamic description of the matter rapidly loses reliability before $1 \mathrm{fm} / c$. 


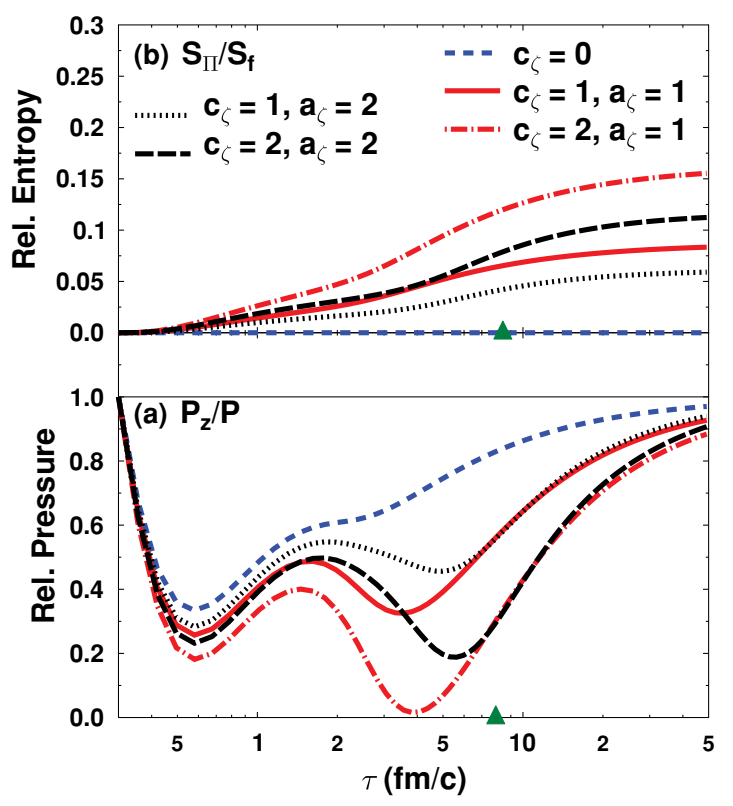

FIG. 3. (Color online) (a) Relative longitudinal pressure $P_{z} / P$ as function of time $\tau$ for scenario (i, S, SYM) and various choices for the bulk viscosity $\left(c_{\zeta}=0,1,2\right.$ and $\left.a_{\zeta}=1,2\right)$. The solid line shows our "standard" parametrization $\left(c_{\zeta}=a_{\zeta}=1\right)$. (b) Relative entropy production from bulk stress, $S_{\Pi} / S_{f}$, as function of time $\tau$ for the same scenarios as in part (a).

We also remind the reader that our results are obtained for the minimal value of the shear viscosity, $\eta=s /(4 \pi)$. Had we chosen a larger shear viscosity, the produced entropy would be respectively larger.

In Fig. 3 we have varied the parameters $c_{\zeta}$ and $a_{\zeta}$, influencing the height and width of the peak in the bulk viscosity near $T_{c}$, respectively, for the scenario (i, S, SYM). The short-dashed (blue) curve in Fig. 3(a) shows the relative longitudinal pressure in the absence of any bulk viscosity for comparison. The solid and dash-dotted (red) lines are obtained for our parametrization of $\zeta(T)$ as shown by the solid (blue) line in Fig. 1, with its height scaled by the factor $c_{\zeta}=1,2$. Obviously, the relative longitudinal pressure drops to almost zero above $T_{c}$ for $c_{\zeta}=2$, indicating that the local equilibrium assumption begins to break down in this case. The dotted and long-dashed (black) curves correspond to the parametrization $\left(a_{\zeta}=2\right)$ for a narrower peak in $\zeta(T)$, as shown by the dashed (blue) line in Fig. 1. In this case, the onset of the strong reduction in $P_{z} / P$ is delayed compared with the case $a_{\zeta}=1$, but the effect in the immediate vicinity of $T_{c}$ [the location is indicated by the triangle on the abscissa] is found to be mainly sensitive to the height of the peak, parametrized by $c_{\zeta}$, not its width. It is important to note, however, that the hydrodynamic evolution becomes increasingly sensitive to the precise value of the equilibration time for the bulk viscosity, $\tau_{\Pi}$, as the width of the peak in $\zeta(T)$ becomes narrower. The small value chosen here $\left(\tau_{\Pi}=\tau_{\pi}\right)$ may be inappropriate for a very narrow peak. Insofar as a narrow, high peak in $\zeta(T)$ is indicative of a near-critical behavior of the medium near $T_{c}$, one would expect any mode that participates in this behavior to exhibit critical slowing down and its relaxation time to increase.

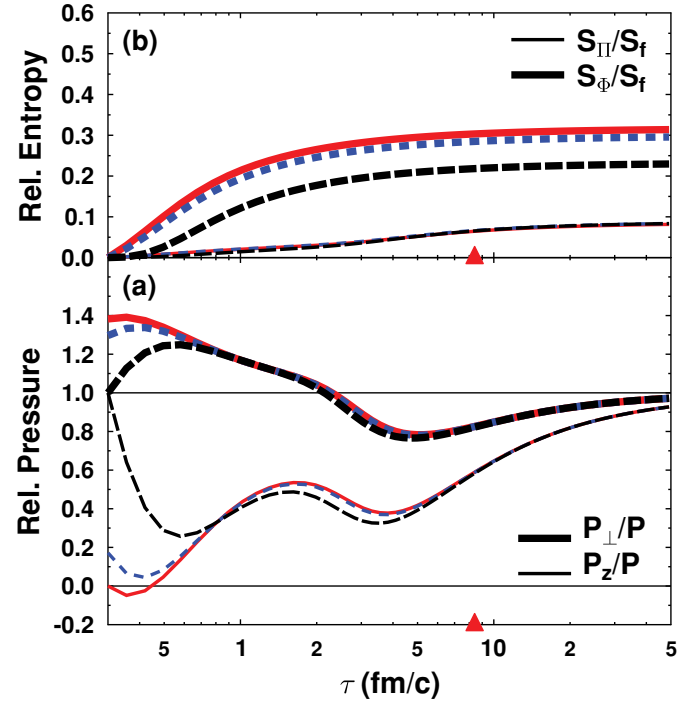

FIG. 4. (Color online) (a) Relative transverse and longitudinal pressure, $P_{\perp} / P$ and $P_{z} / P$, as functions of time $\tau$ for (S, SYM, $c_{\zeta}=1$ ) and initial conditions (i) (black), (ii) (blue), and (iii) (red). (b) Relative entropy production from bulk and shear stress, $S_{\Pi} / S_{f}$ and $S_{\Phi} / S_{f}$, as functions of time $\tau$ for the same set of scenarios. Note that the different curves for $S_{\Pi} / S_{f}$ lie almost on top of each other. The triangle indicates the time of critical temperature for initial condition (iii). For conditions (i) and (ii), $T_{c}$ is reached slightly earlier. All curves are for $a_{\zeta}=1$.

We now compare the impact of different initial conditions on the standard evolution (S) for a bulk viscosity given by the scaling factors $c_{\zeta}=a_{\zeta}=1$ and short relaxation time (SYM). Figure 4(a) shows the relative pressure components $P_{z} / P$ and $P_{\perp} / P$. The most noticeable feature here is that even for equilibrium initial conditions (i), the strong gradients in longitudinal direction drive the system immediately off equilibrium. The first-order initial conditions (ii) are very close to the maximal anisotropic initial conditions (iii), which were loosely extrapolated from classical gluon fields. Interestingly, the effect of the different initial conditions is wiped out after a very short time $\Delta \tau \approx 0.5 \mathrm{fm} / c$, and the system evolves in a universal way from that time forward. However, the different initial conditions for $\Phi$ leave a trace in the entropy produced during this stage of the evolution, as can be seen in Fig. 4(b). On the other hand, the entropy production from bulk stress picks up most contributions around $T_{c}$ and is independent of the initial conditions.

Last, we study the influence of the relaxation times and the conformal terms in the evolution. Figure 5(a) shows $P_{z} / P$ and $P_{\perp} / P$, using the standard equation of motion (S) for $\Phi$ and $c_{\zeta}=a_{\zeta}=1$ using both the estimate for relaxation times $\tau_{\pi}$ and $\tau_{\Pi}$ from kinetic theory (kin), and the lower estimate (SYM). We also show the lower $\tau_{\pi}$ (kin) with the additional conformal terms (C) switched on and $c_{\zeta}=1$, and the same with $c_{\zeta}=0$. Figure 5(b) shows the relative contributions to entropy production in these four scenarios as before.

Obviously, larger relaxation times lead to increased entropy production and larger deviations from equilibrium. This is very clear for the shear contributions at early times. The effect of 


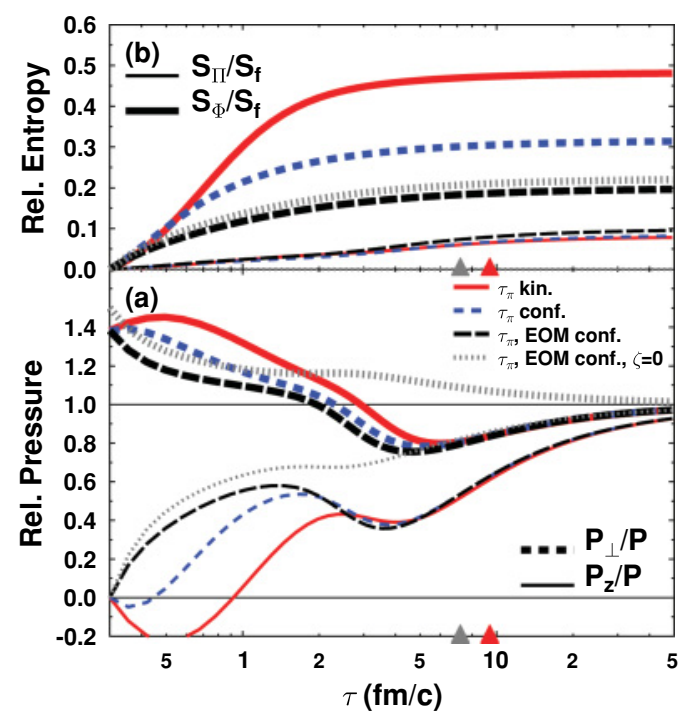

FIG. 5. (Color online) (a) Relative transverse and longitudinal pressure, $P_{\perp} / P$ and $P_{z} / P$. (b) Relative entropy production from bulk and shear stress, $S_{\Pi} / S_{f}$ and $S_{\Phi} / S_{f}$. The different scenarios shown for initial conditions (iii) are $\tau_{\pi}=\tau_{\Pi}=\tau_{\pi}^{(\mathrm{kin})}$ from kinetic theory, see Eq. (11), no conformal terms in Eq. (6), $c_{\zeta}=1$ [red]; $\tau_{\pi}=\tau_{\Pi}=$

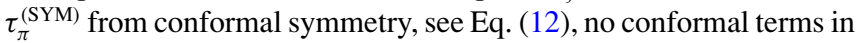
Eq. (6), $c_{\zeta}=1$ [blue]; $\tau_{\pi}=\tau_{\Pi}=\tau_{\pi}^{\text {(SYM) }}$, conformal terms in Eq. (6) switched on, $c_{\zeta}=1$ [black]; $\tau_{\pi}=\tau_{\Pi}=\tau_{\pi}^{(\mathrm{SYM})}$, conformal terms in Eq. (6) switched on, $c_{\zeta}=0$ [gray]. The triangles indicate the largest and smallest times of critical temperature corresponding to the last and the first scenarios, respectively.

different relaxation times seems to be very much suppressed for the bulk stress. In fact, after $\tau \approx 2 \mathrm{fm} / c$, we again see a universal evolution of the system for fixed bulk viscosity. As expected, the additional conformal terms in Eq. (6) lead to a suppression of the shear stress which manifests itself in a rapid relaxation away from the maximum anisotropic initial condition. Enforcing conformal symmetry leads to smaller anisotropies between the transverse and longitudinal directions, and the system is generally closer to equilibrium. This agrees with the recent observation of Song and Heinz [40] made in the context of a study of two-dimensional boost invariant hydrodynamics including transverse expansion.

\section{DISCUSSION AND SUMMARY}

We can now answer some of the questions posed at the beginning of the paper. First, independent of the initial conditions, we find that the longitudinal pressure reaches at most half of the equilibrium pressure throughout the entire lifetime of the quark-gluon plasma phase if the bulk viscosity is close to the values suggested by lattice QCD $\left(c_{\zeta} \approx 1\right)$, even if the shear viscosity takes its minimum value $\eta_{\mathrm{Kss}}$. This keeps the system away from equilibrium and reduces the work done in the longitudinal expansion. Bulk stress is the main contribution to this effect after about $2 \mathrm{fm} / c$, and we find that the evolution of the system after this time, for a given equation of state at equilibrium, is solely determined by the value of the bulk viscosity and largely independent of the initial conditions and relaxation times. Scaling our lattice inspired bulk viscosity with factors $c_{\zeta}>1$ leads to unacceptably small or even negative longitudinal pressure, which would indicate a breakdown of the hydrodynamic picture. These results remain qualitatively unchanged if the width of the peak in the kinematic bulk viscosity is varied by a factor of 2 .

We also found that isotropization of the stress tensor proceeds rather slowly with $\left|P_{T}-P_{z}\right| / P$ as large as $20 \%$ around $T_{c}$. It was recently pointed out by Martinez and Strickland [41] that the anisotropy of the stress tensor of the quark-gluon plasma may be observable via changes in the dilepton yield. On the other hand, the strong reduction in the relative longitudinal pressure, which may even lead to negative values of $P_{z}$ near $T_{c}$, is reminiscent of a first-order phase transition, where the negative pressure is avoided by the formation of a mixed phase. Under favorable conditions, the delay of the expansion caused by mixed phase formation can be observed as a directional dependence of the identicalparticle correlation function in density interferometry [42-44]. The scenario found here may show similar effects, but it differs from the traditional one by the anisotropy of the stress tensor caused by the continued presence of the shear viscosity. A realistic exploration of the influence of the bulk viscosity on identical particle correlations will require the hydrodynamic treatment of the transverse expansion including both bulk and shear viscosities.

On the other hand, we find that bulk stress has a rather modest impact on entropy production, contrary to some previous expectations. For the parametrization $\zeta(T)$ considered here (see Fig. $1, c_{\zeta}=1$ ), the entropy increase due to the bulk viscosity is at most $10 \%$ of the final entropy. The reason is that the velocity gradients due to the longitudinal expansion have decreased significantly when the fireball approaches $T_{c}$. This can also be easily seen by noting that we have only shown bulk and shear stresses relative to the equilibrium pressure. While those are comparable, by the time the maximum in $-\Pi / P$ around $T_{c}$ is reached, the pressure $P$ has dropped significantly and the absolute values of $\Pi$ are much smaller than the values of $\Phi$ reached at times smaller than $1 \mathrm{fm} / c$. A bulk viscosity much larger than that indicated by our extrapolation of the existing lattice QCD results (represented here by scale factors $c_{\zeta} \gg 1$ ) would be necessary to dominate entropy production after decoherence. This statement is independent of the initial condition for the bulk stress.

Nonlinear terms in the evolution of the shear stress, dictated by conformal symmetry, suppress the shear stress and lead to reduced anisotropies and entropy production. However, these effects are not large enough to affect the conclusions drawn above qualitatively.

To summarize, large bulk viscosities around $T_{c}$ lead to prolonged deviations from equilibrium that could be sizable throughout the entire lifetime of the quark-gluon plasma. Bulk viscosities just slightly larger than currently favored could easily lead to a breakdown of the hydrodynamic approximation around $T_{c}$. The decreased pressure should slow down the expansion of the system and increase the time spent in the vicinity of the phase transition. However, the amount of entropy produced through bulk stress around $T_{c}$ is smaller than that produced by shear stress at earlier stages of the evolution and thus does not result in a large increase of the final particle 
multiplicity, unless the bulk viscosity is much larger than that considered here.

\section{ACKNOWLEDGMENTS}

We thank the members of the Yukawa Institute of Theoretical Physics for their hospitality during the YIPQS
Molecule "Entropy Production Before QGP." R.J.F. would like to thank P. Huovinen for helpful discussions. This work was supported in part by the U.S. Department of Energy (Grants DE-FG02-05ER41367, DE-AC02-98CH10886), the RIKEN-BNL Research Center, the Texas A\&M College of Science, and the Bundesministerium für Bildung und Forschung.
[1] I. Arsene et al. (BRAHMS Collaboration), Nucl. Phys. A757, 1 (2005).

[2] B. B. Back et al. (PHOBOS Collaboration), Nucl. Phys. A757, 28 (2005).

[3] J. Adams et al. (STAR Collaboration), Nucl. Phys. A757, 102 (2005).

[4] K. Adcox et al. (PHENIX Collaboration), Nucl. Phys. A757, 184 (2005).

[5] U. W. Heinz and P. F. Kolb, Nucl. Phys. A702, 269 (2002).

[6] P. K. Kovtun, D. T. Son, and A. O. Starinets, Phys. Rev. Lett. 94, 111601 (2005).

[7] T. Hirano and M. Gyulassy, Nucl. Phys. A769, 71 (2006).

[8] T. Hirano, U. W. Heinz, D. Kharzeev, R. Lacey, and Y. Nara, Phys. Lett. B636, 299 (2006).

[9] R. J. Fries, J. Phys. G: Nucl. Part. Phys. 34, S851 (2007).

[10] D. Kharzeev and K. Tuchin, arXiv:0705.4280 [hep-ph].

[11] F. Karsch, D. Kharzeev, and K. Tuchin, Phys. Lett. B663, 217 (2008).

[12] P. Arnold, C. Dogan, and G. D. Moore, Phys. Rev. D 74, 085021 (2006)

[13] P. Arnold, G. D. Moore, and L. G. Yaffe, J. High Energy Phys. 11 (2000) 001.

[14] M. Cheng et al., Phys. Rev. D 77, 014511 (2008).

[15] H. B. Meyer, Phys. Rev. Lett. 100, 162001 (2008).

[16] I. Müller, Z. Phys. 198, 329 (1967).

[17] W. Israel, Ann. Phys. (NY) 100, 310 (1976); W. Israel and J. M. Stewart, Ann. Phys. (NY) 118, 341 (1979).

[18] M. Lublinsky and E. Shuryak, Phys. Rev. C 76, 021901(R) (2007).

[19] G. Torrieri and I. Mishustin, Phys. Rev. C 78, 021901 (2008).
[20] D. H. Rischke and M. Gyulassy, Nucl. Phys. A608, 479 (1996).

[21] J. D. Bjorken, Phys. Rev. D 27, 140 (1983).

[22] G. Baym, Phys. Lett. B138, 18 (1984).

[23] H. Heiselberg and X.-N. Wang, Phys. Rev. C 53, 1892 (1996).

[24] H. Heiselberg and X.-N. Wang, Nucl. Phys. B462, 389 (1996).

[25] D. Teaney, Phys. Rev. C 68, 034913 (2003).

[26] A. Muronga and D. H. Rischke, arXiv:nucl-th/0407114.

[27] A. Muronga, Phys. Rev. Lett. 88, 062302 (2002); 89, 159901(E) (2002).

[28] U. W. Heinz, nucl-th/0512049.

[29] Y. V. Kovchegov and A. Taliotis, Phys. Rev. C 76, 014905 (2007).

[30] A. Muronga, Phys. Rev. C 69, 034903 (2004).

[31] R. Baier, P. Romatschke, and U. A. Wiedemann, Phys. Rev. C 73, 064903 (2006).

[32] R. Baier, P. Romatschke, D. T. Son, A. O. Starinets, and M. A. Stephanov, J. High Energy Phys. 04 (2008) 100.

[33] U. W. Heinz, H. Song, and A. K. Chaudhuri, Phys. Rev. C 73, 034904 (2006).

[34] M. Natsuume and T. Okamura, Phys. Rev. D 77, 066014 (2008).

[35] H. B. Meyer, Phys. Rev. D 76, 101701(R) (2007).

[36] K. Paech and S. Pratt, Phys. Rev. C 74, 014901 (2006).

[37] S. Jeon, Phys. Rev. D 52, 3591 (1995).

[38] B. Müller and A. Schäfer, Phys. Rev. C 73, 054905 (2006).

[39] R. J. Fries, B. Müller, and A. Schäfer, arXiv:0807.1093 [nucl-th].

[40] H. Song and U. W. Heinz, Phys. Rev. C 78, 024902 (2008).

[41] M. Martinez and M. Strickland, arXiv:0805.4552 [hep-ph].

[42] S. Pratt, Phys. Rev. D 33, 1314 (1986).

[43] G. Bertsch, M. Gong, and M. Tohyama, Phys. Rev. C 37, 1896 (1988).

[44] G. F. Bertsch, Nucl. Phys. A498, 173C (1989). 